\title{
Encrypted Vehicular Communication Using Wireless Controller Area Network
}

\author{
Mohammed Al-Qaraghuli ${ }^{1}$, Saadaldeen Rashid Ahmed Ahmed ${ }^{* 2}$, Muhammad Ilyas ${ }^{3}$ \\ ${ }^{1}$ Electrical and Computer Engineering, Altinbas University, Istanbul, Turkey \\ ${ }^{2}$ Computer Science, Tikrit University, Baghdad, Iraq \\ ${ }^{3}$ Electrical and Electronics Engineering, Altinbas University, Istanbul, Turkey
}

\author{
Correspondence \\ *Saadaldeen Rashid Ahmed Ahmed \\ Computer Science, Tikrit University, \\ Baghdad, Iraq \\ Email: Saadaljanabi95@gmail.com
}

\begin{abstract}
In this paper, we focus on ensuring encrypted vehicular communication using wireless controller area network performance at high node densities, by means of Dedicated Short-Range Communication (DSRC) algorithms. We analyses the effect of the vehicular communication parameters, message-rate, data-rate, transmission power and carrier sensing threshold, on the application performance. After a state-of-the-art analysis, we propose a data-rate DSRC algorithm. Simulation studies show that DSRC performs better than other decentralized vehicular communication algorithms for a wide range of application requirements and densities. Vehicular communication plays one of the most important roles for future autonomous vehicle. We have systematically investigated the impact of vehicular communication using the MATLAB ${ }^{\circledR}$ application platform and achieved an accuracy of $93.74 \%$ after encrypting all the communications between the vehicles and securing them by applying the encryption on V2V communication in comparison with the existing system of Sensor Networks which stands at $92.97 \%$. The transmission time for the encryption is 165 seconds while the rate of encryption is as low as 120 Mbps for the proposed awareness range of vehicles to vehicle using DSRC algorithm in Wireless-Controller Area Network for communication. Experimental results show that our proposed method performs 3\% better than the recently developed algorithms.
\end{abstract}

KEYWORDS: Controller area network; Dedicated short-range communication; Encryption; Transmission; V2V; communication;

\section{INTRODUCTION}

In this paper, we have formulated and developed an encrypted vehicular communication using wireless controller area network with introduction of Dedicated Short-Range Communication (DSRC) algorithms. Every year, road traffic accidents kill about 1.3 million people worldwide, and severely injure another 50 million [1]. Nearly 33,000 [2] and 27,000 [3] deaths happen every year due to road traffic accidents in US and Europe respectively. There were around 3500 road fatalities in Turkey in 2015 [4]. The estimated economic loss due to road traffic accidents for the Turkey is over 7 billion euros every year.

Encrypted and Advanced Driver Assistance Systems (EADAS) have been developed, to alleviate the burden on drivers and improve driving safety [5]. EADAS makes the driver aware of potentially hazardous situations in the environment and instruct the driver to take corrective actions. In some cases, corrective actions are taken automatically, i.e., without any assistance from the driver.
These are known as automation systems [6]. EADAS and self-driving cars can use the surrounding connected vehicles to sense the environment. Connected vehicles add the following benefits to systems that only rely on sensors [7]. Connected vehicles provide an extended field of perception, beyond line-of-sight, and hence, allow the detection of threats invisible to on-board sensors.

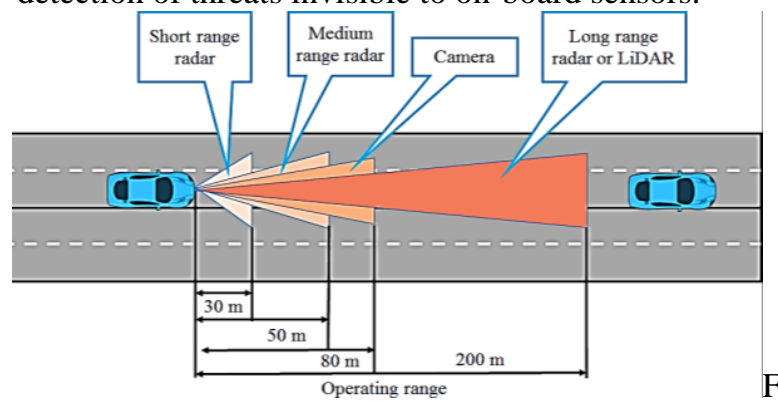

Fig.

1. Sensor operation range and line-of-sight detection [7]

The sensing encrypted range of a vehicle is defined as the range around a vehicle within which the vehicle senses the channel busy if other vehicles in the range transmit the 
message [8]. Vehicles sense each other message transmissions to avoid message collisions. Hidden nodes of a vehicle (node) are vehicles that do not sense each other message transmissions but they can sense the transmissions of the vehicle [9]. Hidden nodes may lead to message collisions, as they cannot sense each other's transmissions. The hidden node problem is common in carrier sensing mechanism based wireless networks. Capture effect can reduce the hidden node problem in $\mathrm{V} 2 \mathrm{X}$ communication.

\section{A. Problem Statement}

Vehicular Safety applications track the neighbor vehicles to predict and avoid dangerous situations. There are different problems and challenges that must need an attention to be solved in the first place. Some of the problems and challenges are given by:

- The effects of unreliable communication on application reliability

- Less secure communication

- Limited channel capacity

- Dynamic vehicular environment

- Broadcast transmission

- Low sensing range of vehicle

B. Research Contribution

Optimizing the usage of the encrypted channel so that vehicular communication applications are sustained even at large vehicular densities, is crucial. The objective of this paper is to develop an encrypted vehicular communication system using wireless CAN with DSRC algorithm and to ensure reliable safety application performance at high vehicular densities by means of DSRC algorithms. Specifically, data-rate adaptation techniques are explored to make DSRC algorithms scalable to high vehicular densities. The specific questions we address in this paper are the following:

R1: To what extent communication parameters influence vehicular communication performance?

R2: To what extent various DSRC algorithms with wireless CAN influence application performance?

R3: To what extent does the data-rate DSRC with wireless CAN improve the application performance compared to DSRC algorithms reported in the literature?

R4: To what extent coexistence of new DSRC algorithms with the already deployed DSRC affects the application performance?

R5: Can we experimentally validate the results of DSRC algorithms for encrypted vehicular communication?

The positioning of vehicles is performed using Global Navigation Satellite Systems (GNSS) such as GPS (Global Positioning System) and Galileo [10]. Accurate positioning of the vehicles is necessary for reliable TTC estimation [11]. The VSC-A project [12] by USDOT identified two different levels of GPS accuracy, for different classes of safety applications: road-level and lane-level. Applications such as EEBL require a roadlevel accuracy of less than $5 \mathrm{~m}$ and applications such as FCW require a lane-level accuracy of less than $1.5 \mathrm{~m}$ [13]. Vehicular communication has investigated GPS accuracy and availability at various urban, rural, and highway environments. It concluded that GPS is adequate in most of the environments [14]. Although GPS outage may appear in deep urban environments [15], techniques that estimate the position of a vehicle based on in-vehicle sensors information such as speed and yaw rate can be utilized. Research is ongoing to improve the availability and accuracy of GPS.

\section{Runtime adaptation of DSRC based on channel quality}

Due to the highly dynamic vehicular environment the channel quality changes due to shadowing and scenarios such as rural and urban affecting the packet reception ratio PRR, i.e., probability of beacon message delivery [16]. Changes in PRR may affect the application performance. Thus, to ensure the reliability of applications DSRC algorithms should adapt parameters, such as the minimum required message-rate of the application, based on the channel quality on runtime [17]. DSRC algorithms such as SAE [18] use PRR as channel quality indicator and generate additional beacon messages when the PRR decreases below a threshold to ensure reliability [19]. We recommend the investigation of such DSRC adaptation mechanisms further. Accurate positioning of the vehicles is necessary for reliable DSRC estimation. The PRC project for vehicles [20] by USDOT identified two different levels of GPS accuracy.

\section{Data transmit rate of DSRC}

The benefit of DSRC is that it guarantees the awareness range requirement of the application. DSRC tries to maximize the awareness range for a fixed transmit power. However, applications have a minimum awareness range requirement [21]. Thus, tuning the transmit power to guarantee the desired awareness range along with message-rate and data-rate might further increase the application performance [22]. The required transmit power for a desired awareness range changes with datarate. Further studies are necessary to analyze the impact of tuning the data-rate on the selection of the transmit power and vice-versa. We recommend the investigation of DSRC algorithms that tune multiple times the same parameters as they can improve the maximum vehicular density supported by the channel.

$$
T_{D}=\left\{\exp \left[\frac{v-v_{\max }}{v_{\max }}\right]\right\}, \text { for } v \leq v_{\max }
$$

Where $T_{D}$ is the data transmission rate, ' $v$ ' represents the velocity of vehicle and $v_{\max }$ represents the maximum velocity of vehicle with respect to the data transmission as they are taken from [22]. We have discussed ways to 
measure the application reliability, which reflects the effect of unreliable wireless communication on the reliability of the application [23]. The application requirements have been mapped to communication parameter requirements, in particular, the minimum message-rate, and PRR to ensure reliable safety applications [24]. We have shown that increasing the message-rate can increase the application reliability [25]. The analysis of this chapter will be utilized in the forthcoming chapters to design an efficient congestion control algorithm that can guarantee the reliable operation of safety applications [26].

We presented a preliminary model to analyze the effect of the PRR and message-rate on application reliability. Further analysis of the application reliability considering the mobility of vehicles, channel characteristics and scenarios should be part of a future study [27].

\section{E. V2X Communication Reliability}

V2X safety application reliability is determined by several subsystems as shown in Fig 2 [28]. The sensor subsystem gathers sensor information such as position, velocity and acceleration. The communication subsystem transmits its neighbor vehicles sensor information to an application controller using V2X communication. The application controller computes the safety metric such as TTC to decide the necessary actions, which are then performed by the actuator subsystem. In awareness warning phase the warnings are provided to the driver to decide the necessary actions; however, in automatic pre/post-crash the system decides the necessary actions such as automatic braking, steering and safety system deployment such as air bags. We have analyzed the time delay for propagating the transmission messages represented by ' $d$ ' which is defined as the difference between the timestamps of message reception $t_{R x}$ and transmission $t_{T x}$ respectively by equation (2) taken from [25].

$$
d=t_{R x}-t_{T x}
$$
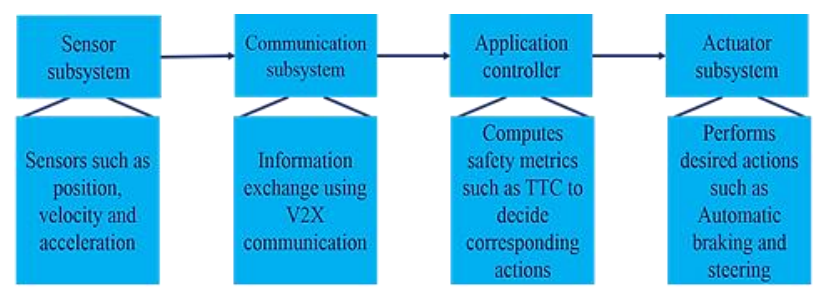

Fig. 2. Function sequence of V2X safety application [28].

\section{F. V2X Communication Application}

Tuning the communication parameters may affect the application reliability and awareness range of the application. Message-rate based DSRC algorithms in CAN network may decrease the message-rate below the minimum required message-rate to avoid congestion affecting the application reliability [29]. Transmit power and carrier sensing threshold DSRC algorithms limit the transmission and sensing range respectively which may conflict with the awareness range requirements of the application. Similarly, data-rate DSRC algorithms with wireless CAN limit the communication range which may affect the awareness range requirements of the application

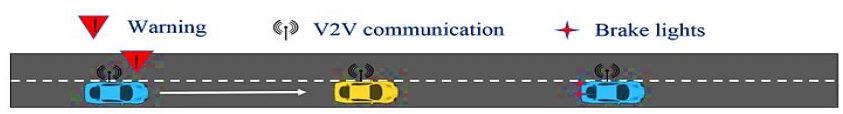

(a) Emergency electronic brake light

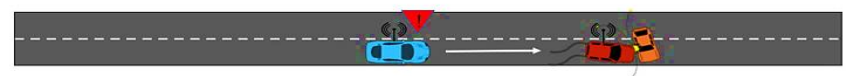

(b) Pre-crash warning

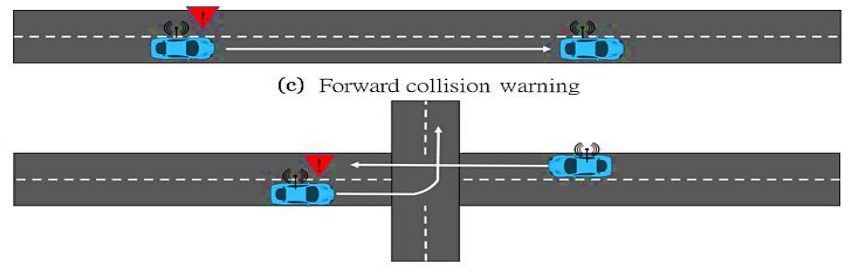

(d) Left turn assistant

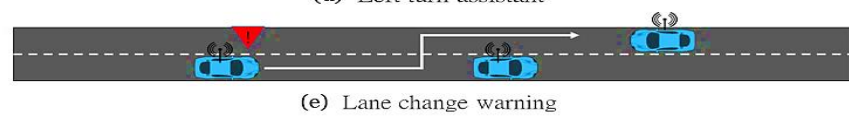

Fig. 3. Different types of V2X communication with warnings. [30]

The general principle of V2X safety applications, e.g. encrypted V2V applications suggested by the USDOT VSC project as shown in Fig. 3 [30], is to use the exchanged information among vehicles to compute a safety metrics. DSRC algorithms should choose the appropriate communication parameters such that they avoid congestion and simultaneously satisfy the minimum application reliability and awareness range requirements of the application.

\section{METHODOLOGY}

In this research work, to encrypt the communication of vehicles we proposed a DSRC algorithm in wireless CAN network for a large number of vehicles, we would need a large number of wireless CAN networks in the open-source dataset as well as vehicular communication data present in dataset. In order to encrypt the communication of vehicles, we utilize an emulation platform MATLAB where each wireless CAN emulates multiple vehicles.

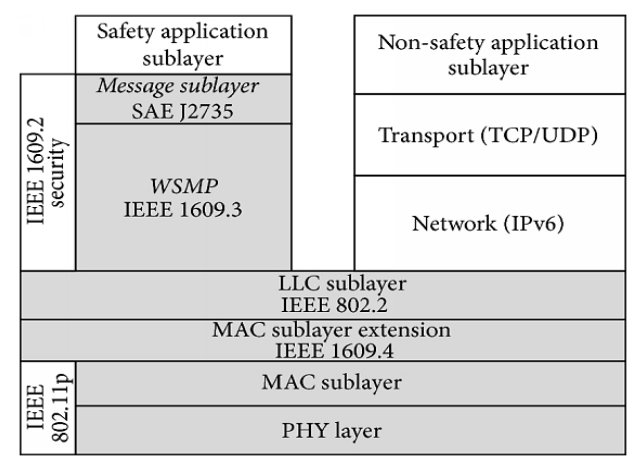

Fig. 4. DSRC architecture specified by Network. 
We are interested in the behavior of the DSRC algorithms for large vehicular densities. Since in reality each vehicle has its own CAN network operating independently from other CAN network, we would, in principle, need a number of CAN network in the order of three thousand. Since this is infeasible due to cost reasons, we chose to emulate multiple virtual CAN networks on one physical CAN network. We call a physical CAN network an Emulation Node (EN), i.e., a device that emulates the behavior of multiple CAN networks. The number of physical layer devices we had available was four. Each EN generates and transmits several beacon messages as if generated by many CAN network (several hundred vehicles). Of course, our emulation approach deviates from the behavior of CAN networks in real traffic scenarios. We examine what this means, and consequently what the system limitations are of the emulation platform and the impact on the behavior of wireless CAN networks, while leaving this out has no influence on the performance aspects of DSRC we are present the propagation delay in equation (3) however the we propose finding the respective coordinates of vehicles in equation (4). In a similar way, we define message delivery time for $\mathrm{V} 2 \mathrm{~V}$ communications as:

$$
d=d+\Delta T
$$

where $\mathrm{d}[\mathrm{s}]$ is the propagation time delay within a cluster, as defined by [31], and $\Delta \mathrm{T}[\mathrm{s}]$ is the minimum time interval

$$
D_{S D}=\sqrt{\left(y_{D}-y_{S}\right)^{2}+\left(X_{D}-X_{S}\right)^{2}}
$$

where $(\mathrm{xS}, \mathrm{yS})$ and $(\mathrm{xD}, \mathrm{yD})$ are the respective coordinates of vehicle $S$ and vehicle D [30].

\section{DSRC Algorithm in Wireless CAN}

Many devices support wireless CAN and have a DSRC architecture specified by Network [31]. We adopt this DSRC architecture, shown in Fig. 4, where the interaction between the DSRC algorithm and the protocol layers in network are shown. This is similar to the network architecture. The PHY, MAC and LLC layers of IEEE 802.11p in wireless CAN network are accessed via the Logical Link Control Application Programming Interface (LLC-API). The application and facilities layer of network are accessed using the Application Facilities Layer Application Programming Interface (AF-API) in MATLAB. The application layer runs a pseudo application to generate the beacon

Messages. The facilities layer controls the messagerate as determined by the DSRC algorithm. The focus of the experiments is on the broadcast transmission of beacon messages and DSRC algorithms in which the network and transport layers are not involved, hence, these are not considered in our implementation. Note that in our implementation, we leave out security and privacy of beacon messages. Implementing this would require a lot of processing by the safety devices, which would seriously restrict the number of OBUs [32] that can be emulated by an EN, while leaving this out has no influence on the performance aspects of DSRC we are interested in. The DSRC algorithms obtain the channel load information, CAN network and packet count, sensed by the physical layer via the MATLAB and they adjust the message-rate, data-rate, and transmit power accordingly.

TABLE I

PROPOSED AWARENESS RANGE OF DIFFERENT VEHICLES USING DSRC ALGORITHM IN WIRELESS CAN FOR COMMUNICATION IN COMPARISON WITH DIFFERENT TECHNIQUES

\begin{tabular}{c|ccccc}
\hline \hline Parameters & \multicolumn{5}{|c}{ Approach awareness range (m) } \\
\cline { 2 - 6 } & $\begin{array}{c}\text { Wirel } \\
\text { ess } \\
\text { CAN }\end{array}$ & $\begin{array}{c}\text { IP- } \\
\text { SEC } \\
{[23]}\end{array}$ & $\begin{array}{c}\text { Sensor } \\
\text { Networ } \\
\text { ks [23] }\end{array}$ & $\begin{array}{c}\text { PDR- } \\
\text { DCC } \\
{[23]}\end{array}$ & $\begin{array}{c}\text { LIMER } \\
\text { IC [23] }\end{array}$ \\
\hline $\begin{array}{c}\text { Encryption } \\
\text { Transmission } \\
\text { Time (Overall) }\end{array}$ & 165 & 180 & 175 & 195 & 175 \\
\hline $\begin{array}{c}\text { Rate of } \\
\text { Encryption } \\
\text { (Overall) }\end{array}$ & 120 & 145 & 140 & 155 & 125 \\
\hline \hline
\end{tabular}

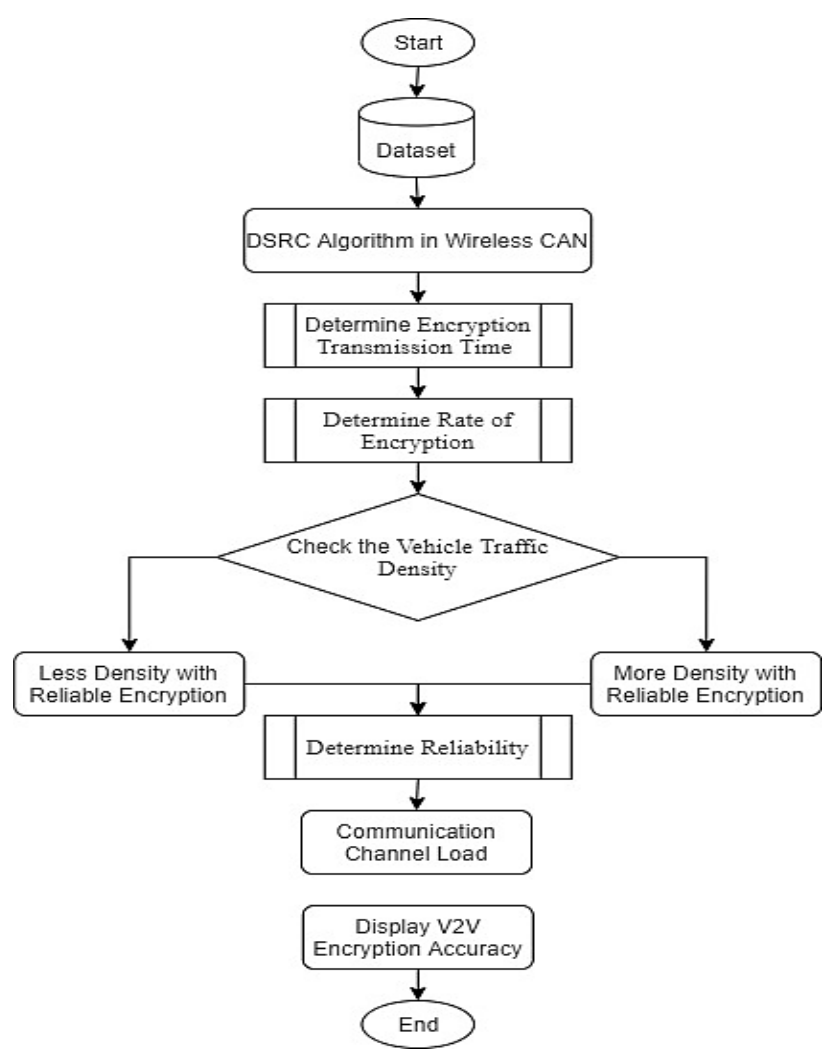

Fig. 5. The pictorial representation of followed approach.

D. Encryption in Wireless Controller Area Network (CAN)

An encrypted vehicular communication emulates multiple wireless CAN networks; however, it has a single PHY and MAC protocol entity. In reality, the wireless CAN's belonging to different vehicles generate their messages independently and use their own MAC entity to access the 
channel, competing with the other wireless CANs. Since, in case of contention, the MAC protocol uses random back-off, the order in which messages finally access the channel is also random [33]. In our new emulation experiments, however, messages generated by multiple wireless CANs, emulated by the same DSRC algorithm for encryption, queue up in the same MAC layer queue, and hence try to access the channel one after the other, in the order in which they were generated. The implications of this are:

1. There is no contention between messages generated by the same DSRC algorithm emulating several wireless CAN networks. Thus, there are no collisions between the messages from wireless CAN emulated by the same DSRC algorithm. In reality encryption occur. On the other hand, messages generated by different wireless CAN, still compete. In brief, the packet reception ratio (PRR) experienced by the emulated vehicles is better than in reality. Further study is necessary to quantify the effect of the emulation platform on DSRC performance.

2. Since the encryption messages emulated by the same wireless CAN access the channel sequentially, the channel access time is increased. This effect is discussed in detail in [34]. The study concludes that the increased channel access time limits the maximum channel load that can be created by the emulation platform. Furthermore, the study shows that augmenting the beacon size increases the maximum channel load attainable by the emulation platform.

TABLE II

PROPOSED AWARENESS RANGE OF DIFFERENT VEHICLES USING DSRC ALGORITHM IN WIRELESS CAN FOR COMMUNICATION WITH DENSITY.

\begin{tabular}{c|c|ccc}
\hline \hline \multirow{4}{*}{ Parameters } & \multirow{2}{*}{\begin{tabular}{c} 
Vehicle \\
\cline { 3 - 5 }
\end{tabular}} & \multicolumn{4}{|c}{ Approach awareness range (m) } \\
\cline { 3 - 5 } & Traffic & Wireless & IP-SEC & Sensor \\
& Density & CAN & {$[23]$} & $\begin{array}{c}\text { Networks } \\
{[23]}\end{array}$ \\
\hline Encryption Transmission & & & & 150 \\
Time & Less Density & 78 & 160 & 150 \\
Rate of Encryption & More Density & 126 & 150 & 100 \\
& Less Density & 1 & 5 & 3 \\
& More Density & 1 & 8 & 5 \\
\hline \hline
\end{tabular}

\section{Encrypting the Communication}

In vehicular communication networks, the elapsed time in receiving the controller area network (CAN) bus information from the CAN network of the vehicle should be taken into consideration, prior to the transmission of data to the other vehicle. Once the data is transmitted either by IEEE802.11p or LTE, Time of Flight (TOF) comes into picture [35]. To estimate the overall performance of vehicular communication network these two-time factors need to be considered. A mobile service can be used to provide the live updates of the traffic situation on the road to the users. To get the live updates, the users need to transmit and receive the data from the remote server when they are driving on the road. We determine the performance of IEEE802.11p and LTE as vehicular networks in real time. Each wireless CAN network emulates a quarter of the total number of vehicles. In our preliminary experiments, the wireless CAN transmit at the default $10 \mathrm{~Hz}$ message-rate without any DSRC algorithm. The maximum data-rate of the emulation platform is fixed at $18 \mathrm{Mbps}$. For each vehicular communication and data-rate, we run the experiment for $60 \mathrm{~s}$ on MATLAB. We average measurements of all four wireless CAN networks using DSRC algorithm

Table III

PARAMETERS OF DIFFERENT MEASUREMENTS FOR ENCRYPTED VEHICULAR COMMUNICATION.

\begin{tabular}{c|c}
\hline \hline Parameters & Measurements \\
\hline Encrypted Data Size (Payload) & 500 bytes \\
Channel load threshold (CBPT) & $60 \%$ \\
Vehicle transmit power & $0 \mathrm{dBm}$ \\
Peak antenna gain & $4.6 \mathrm{dBi}$ \\
Vehicle carrier sensing threshold & $-95 \mathrm{dBm}$ \\
Message-rate & 1 to $10 \mathrm{~Hz}$ \\
Data-rate [11] & $3,4.5,6,9,12$, and $18 \mathrm{Mbps}$ \\
Total Vehicular Communication & $5.9 \mathrm{GHz}$ \\
\hline \hline
\end{tabular}

1) Collecting the vehicle information in real time and transmitting the information to a remote TCP (Transmission Control Protocol) server or UDP (User Datagram Protocol) server.

2) Calculating the time elapsed to receive controller area network (CAN) data from the CAN network and transmitting to the server at different traffic situations and driving speeds. The elapsed time in transmitting the user data to the server is an essential challenge to estimate the overall performance of the vehicular networks.

\section{RESULTS}

Encrypted vehicular communications System utilizes dynamics information provided by the vehicle to estimate the driver's intended future path. The estimate is provided without dependence on future road geometry information obtained from outside sources (e.g., map databases, vehicle probes). In order to determine if the LTE can meet the minimum vehicular communication requirement, the first step that has been considered to determine the latency. The MATLAB is being used for the simulation purpose with several built-in libraries. Due to mobility, vehicles move in and out of the observing zone. Thus, the vehicles and the links (sender-receiver pairs) on which the data is encrypted change over time. 


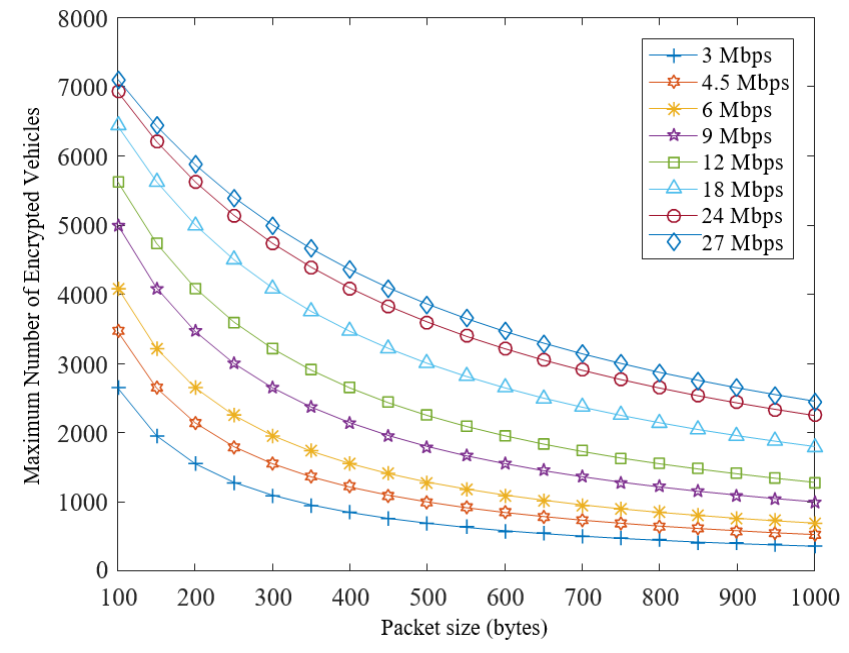

Fig. 6. Encrypted transmission time as a function of packet size for different data-rates during communication using DSRC algorithm.

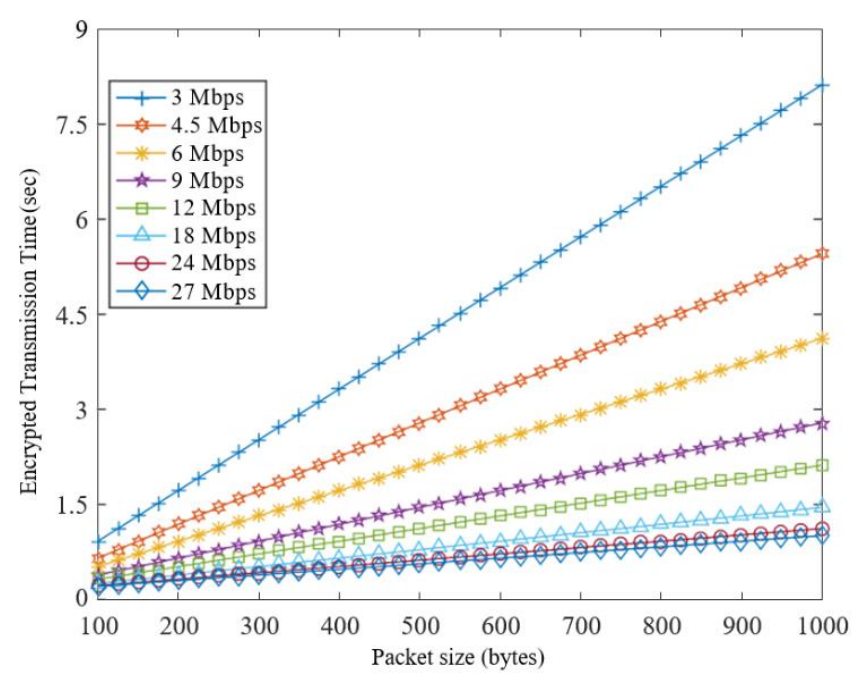

Fig. 7. Maximum number of encrypted vehicles per second in the channel for different data-rates and packet sizes for secure communication using DSRC algorithm

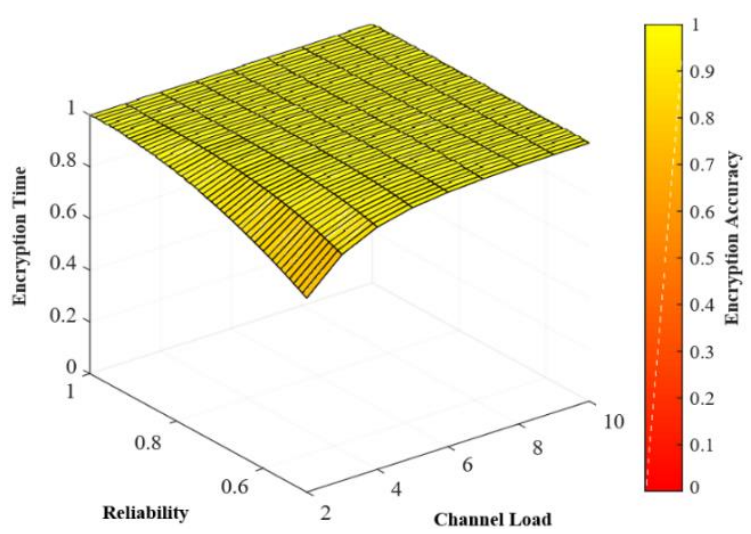

Fig. 8. For best case vehicular communication encryption, the encryption time with reliability on y-axis however the encryption accuracy with channel load stands at $93.74 \%$.

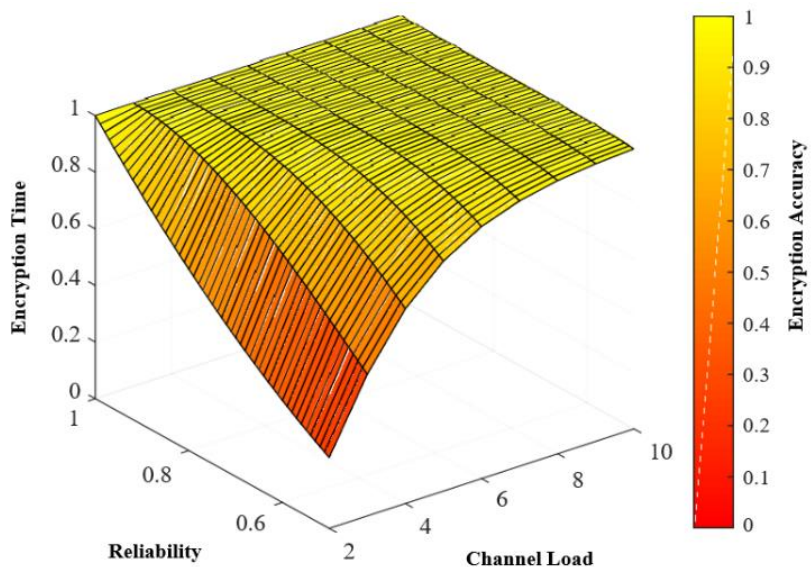

Fig. 9. For worst case vehicular communication encryption, the encryption time with reliability on y-axis however the encryption accuracy with channel load stands at $47.89 \%$.

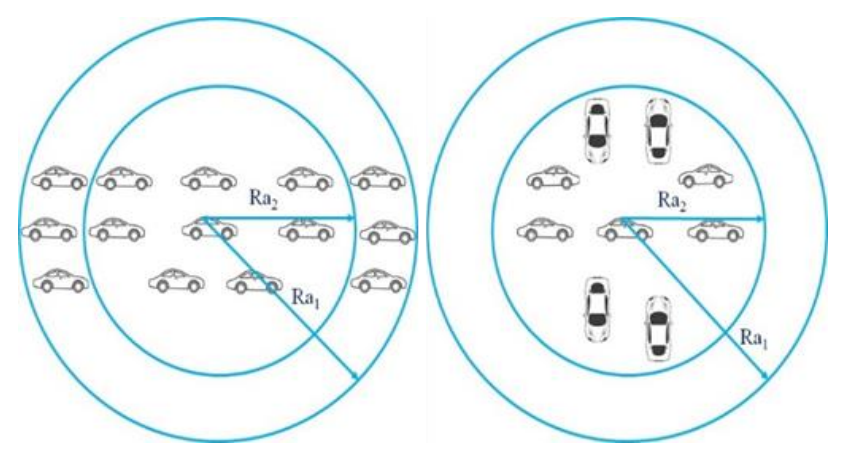

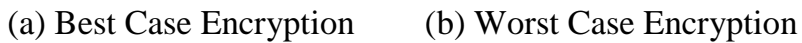
Fig. 10: Pictorial demonstration of encrypting the vehicular communication in both best and worst case.

Over a simulation time of $60 \mathrm{~s}$, we collect data on 600 to 800 vehicles and 900,000 to $1,500,000$ links presents in dataset passing in the MATLAB as library were encrypted for communication. The specific number of vehicles and links depends on the vehicular density and the DSRC algorithm.

\section{DISCUSSION}

Vehicular communication plays one of the important roles for future autonomous vehicle. Current vehicular communication is more directed towards DSRC (Dedicated Short-Range Communications). The DSRC law is not enforced and some other technology such as D2D (Device to Device) based on LTE (Long Term Evolution) will provide good results and become possible alternative using the wireless controller area network for communication. The scope of this paper is to have an encryption based DSRC algorithm with wireless CAN network that has the capability to process transmit and receive the data and could able to interface all of the following devices for vehicular communication. And also, to have a capability to help in the future of autonomous vehicles. We have compared the accuracy of wireless controller area network that encrypts vehicle-to- 
vehicle communication with existing techniques that are being used for encryption of communication in [23].

\section{TABLE IV}

THE COMPARISON OF WIRELESS CAN NETWORK WITH OTHER EXISTING DEVELOPED SYSTEMS FOR ENCRYPTION

\begin{tabular}{c|ccc}
\hline \hline \multicolumn{2}{c}{ LIMERIC [23] } & \multicolumn{2}{c}{ PDR-DCC [23] } \\
\hline Message-rate & 1 to $10 \mathrm{~Hz}$ & Message-rate & $10 \mathrm{~Hz}$ \\
Data-rate & $6 \mathrm{Mbps}$ & Data-rate & $3,4.5,6,9$, \\
& & & 12 and 18 \\
& & Mbps \\
Encryption & $92.45 \%$ & Encryption & $79.56 \%$ \\
Accuracy & & Accuracy & \\
\hline
\end{tabular}

\begin{tabular}{c|ccc}
\hline \multicolumn{2}{c}{ Sensor Networks [23] } & \multicolumn{2}{c}{ Wireless CAN } \\
\hline Message-rate & 1 to $10 \mathrm{~Hz}$ & Message-rate & 1 to $10 \mathrm{~Hz}$ \\
Data-rate & $3,4.5,6,9$, & Data-rate & $6 \mathrm{Mbps}$ \\
& $\begin{array}{c}12 \text { and } 18 \\
\text { Mbps }\end{array}$ & \\
& $89.56 \%$ & $\begin{array}{c}\text { Encryption } \\
\text { Accuracy }\end{array}$ & $93.74 \%$ \\
Encryption & & Accuracy &
\end{tabular}

This research paper explains the research study, done for one of the performance evaluations of encrypted vehicular communication using wireless controller area network. It can be further continued to develop a device and a server with more functionalities for encrypted vehicular communication.

\section{CONCLUSION}

In this paper, we have developed an encrypted vehicular communication using wireless controller area network with the help of DSRC encryption algorithm. Vehicular communication-based safety applications rely on exchange of messages between vehicles, to inform their environment, and, foresee and avoid hazardous situations. We focused on ensuring a desirable encryption performance at high vehicular densities by means of Dedicated Short-Range Communication (DSRC) algorithms. Channel load is the major cause for degradation of the encryption performance at high vehicular densities. DSRC algorithms optimize the usage of the channel to avoid load which is crucial for a desirable encryption performance at high vehicular communication. We have systematically investigated the effect of vehicular communication on the MATLAB application platform and achieved an accuracy of $93.74 \%$ for encrypting all the communications between the vehicles and making them secure in symmetry. The encryption transmission time for the encryption recorded at 165 seconds while the rate of encryption recorded as low as 120 Mbps for the proposed awareness range of different vehicles using DSRC algorithm in wireless CAN for secure vehicular communication.

\section{CONFLICT OF INTEREST}

The authors have no conflict of relevant interest to this article.

\section{REFERENCES}

[1] E. Halawany, B.M.; Jameel, F.; Da Costa, D.B.; Dias, U.S.; Wu, K. Performance Analysis of Downlink NOMA Systems over $\kappa-\mu$ Shadowed Fading Channels. IEEE Trans. Veh. Technol. 2019, 69, 1046-1050

[2] K. Anwar, W.; Franchi, N.; Fettweis, G. Performance Evaluation of Next Generation V2X Communication Technologies: 5G NR-V2V vs. IEEE 802.11bd. In Proceedings of the IEEE $90^{\text {th }}$ Vehicular Technology Conference Vehicular Technology Conference (VTC-Fall 2019), At Honolulu, HI, USA, 22-25 September 2019

[3] L. Magueta, R.; Teodoro, S.; Castanheira, D.; Silva, A.; Dinis, R.; Gameiro, A. Multiuser Equalizer for Hybrid Massive MIMO mmWave CE-OFDM Systems. Appl. Sci. 2019, 9, 3363

[4] L. Magueta, R.; Castanheira, D.; Silva, A.; Dinis, R.; Gameiro, A. Hybrid multi-user equalizer for massive MIMO millimeter-wave dynamic subconnected architecture. IEEE Access 2019, 7, 79017-79029.

[5] D. Castanheira, D.; Lopes, P.; Silva, A.; Gameiro, A. Hybrid beamforming designs for massive MIMO millimeter-wave heterogeneous systems. IEEE Access 2017, 5, 21806-21817

[6] F. Magueta, R.; Castanheira, D.; Silva, A.; Dinis, R.; Gameiro, A. EADAS iterative space-time equalization for multi-user mmW massive MIMO systems. IEEE Trans. Commun. 2016, 65, 608-620

[7] L. Jameel, F.; Haider, M.A.A.; Butt, A.A. Second order fading statistics of UAV networks. In Proceedings of the 2017 Fifth International Conference on Aerospace Science \& Engineering (ICASE), Islamabad, Pakistan, 1416 November 2017; pp. 1-6

[8] L. Jameel, F.; Jabeen, F.; Hamid, Z. Analysis of cochannel interference in VANETs under nakagami-m fading. In Proceedings of the 2016 International Conference on Frontiers of Information Technology (FIT), Islamabad, Pakistan, 19-21 December 2016; pp. 153-158 [9] M. Khan, F.; Pi, Z. mmWave mobile broadband (MMB): Unleashing the $3-300 \mathrm{GHz}$ spectrum. In Proceedings of the 34th IEEE Sarnoff Symposium, Princeton, NJ, USA, 3-4 May 2011; pp. 1-6

[10] L. Jameel, F.; Haider, M.A.A.; Butt, A.A. Robust localization in wireless sensor networks using RSSI. In Proceedings of the 2017 13th International Conference on Emerging Technologies (ICET), Islamabad, Pakistan, 2728 December 2017; pp. 1-6

[11] L. Giordani, M.; Mezzavilla, M.; Dhananjay, A.; Rangan, S.; Zorzi, M. Channel Dynamics and SNR Tracking in Millimeter Wave Cellular Systems. In Proceedings of the 22th European Wireless Conference, VDE Association, Oulu, Finland, 18-20 May 2016; pp. 306-313 
[12] E. Verdone, R. Outage probability analysis for shortrange communication systems at $60 \mathrm{GHz}$ in ATT urban environments. IEEE Trans. Veh. Technol. 2017, 46, 10271039

[13] Y. Wang, Y.; Venugopal, K.; Heath, R.W.; Molisch, A.F. MmWave vehicle-to-infrastructure communication: Analysis of urban microcellular networks. IEEE Trans. Veh. Technol. 2018

[14] M. Petrov, V.; Kokkoniemi, J.; Moltchanov, D.; Lehtomaki, J.; Juntti, M.; Koucheryavy, Y. The Impact of Interference from the Side Lanes on mmWave/THz Band V2V Communication Systems with Directional Antennas. IEEE Trans. Veh. Technol. 2018, 67, 5028-5041

[15] [15] L. Milanés, V.; Shladover, S.E.; Spring, J.; Nowakowski, C.; Kawazoe, H.; Nakamura, M. Cooperative Adaptive Cruise Control in Real Traffic Situations. IEEE Trans. IEEE Trans. Intell. Transp. Syst. 2014, 15, 296-305. [16] K. Karagiannis, G.; Altintas, O.; Ekici, E.; Heijenk, G.; Jarupan, B.; Lin, K.; Weil, T. Vehicular Networking: A Survey and Tutorial on Requirements, Architectures, Challenges, Standards and Solutions. IEEE Commun. Surv. Tutorials 2016, 13, 584-616

[17] P. Kim, B.; Yi, K.; Yoo, H.-J.; Chong, H.-J.; Ko, B. An IMM/EKF Approach for Enhanced Multitarget State Estimation for Application to Integrated Risk Management System. IEEE Trans. Veh. Technol. 2015, 64, 876-889

[18] F. Zingoni, A.; Diani, M.; Corsini, G. A Flexible Algorithm for Detecting Challenging Moving Objects in Real-Time within IR Video Sequences. Remote Sens. 2017, 9, 1128

[19] D. Ponte Müller, F.; Diaz, E.M.; Rashdan, I. Cooperative Positioning and Radar Sensor Fusion for Relative Localization of Vehicles. In Proceedings of the 2016 Intelligent Vehicles Symposium (IV), Gothenburg, Sweden, 19-22 June 2016; pp. 1060-1065

[20] R. Kloiber, B.; Strang, T.; Röckl, M.; de Ponte Müller, F. Performance of CAM Based Safety Applications Using ITS-G5A MAC in High Dense Scenarios. In Proceedings of the 2011 Intelligent Vehicles Symposium (IV), BadenBaden, Germany, 5-9 June 2016; pp. 654-660

[21] E. Liu, J.; Wan, J.; Wang, Q. A survey on positionbased routing for vehicular ad hoc networks. J. Telecommun. Syst. Arch. 2016, 62, 15-30.

[22] J. Lin, Y.W.; Chen, Y.S.; Lee, S.L. Routing Protocols in Vehicular Ad Hoc Networks: A Survey and Future Perspectives. J. Inf. Sci. Eng. 2010, 26, 913-932

[23] Y. Lee; M. Müller. Survey of Routing Protocols in Vehicular Ad Hoc Network Communication for Encryption. In Advances in Vehicular Ad-Hoc Networks: Developments and Challenges; Information Science Reference (an Imprint of IGI Global); United States of America: New York, NY, USA, 2009; pp. 113-175

[24] Y. Bilal, S.M.; Khan, A.R.; Ali, S. Review and performance analysis of position-based routing protocols. Wirel. Pers. Area Commun. 2016, 1, 559-578

[25] E. Halawany, B.M.; Jameel, F.; Da Costa, D.B.; Dias, U.S.; Wu, K. Performance Analysis of Downlink NOMA
Systems over $\kappa-\mu$ Shadowed Fading Channels. IEEE Trans. Veh. Technol. 2019, 69, 1046-1050

[26] T. Anwar, W.; Franchi, N.; Fettweis, G. Performance Evaluation of Next Generation V2X Communication Technologies: 5G NR-V2V vs. IEEE 802.11bd. In Proceedings of the IEEE 90th Vehicular Technology Conference (VTC-Fall 2019), At Honolulu, HI, USA, 2225 September 2019.

[27] R. Magueta, R.; Teodoro, S.; Castanheira, D.; Silva, A.; Dinis, R.; Gameiro, A. Multiuser Equalizer for Hybrid Massive MIMO mmWave CE-OFDM Systems. Appl. Sci. 2019, 9, 3363

[28] R. Magueta, R.; Castanheira, D.; Silva, A.; Dinis, R.; Gameiro, A. Hybrid multi-user equalizer for massive MIMO millimeter-wave dynamic subconnected architecture. IEEE Access 2019, 7, 79017-79029

[29] E. Castanheira, D.; Lopes, P.; Silva, A.; Gameiro, A. Hybrid beamforming designs for massive MIMO millimeter-wave heterogeneous systems. IEEE Access 2017, 5, 21806-21817

[30] T. Magueta, R.; Castanheira, D.; Silva, A.; Dinis, R.; Gameiro, A. Hybrid iterative space-time equalization for multi-user mmW massive MIMO systems. IEEE Trans. Commun. 2016, 65, 608-620

[31] U. Jameel, F.; Haider, M.A.A.; Butt, A.A. Second order fading statistics of UAV networks. In Proceedings of the 2017 Fifth International Conference on Aerospace Science \& Engineering (ICASE), Islamabad, Pakistan, 1416 November 2017; pp. 1-6

[32] U. Jameel, F.; Jabeen, F.; Hamid, Z. Analysis of cochannel interference in VANETs under nakagami-m fading. In Proceedings of the 2016 International Conference on Frontiers of Information Technology (FIT), Islamabad, Pakistan, 19-21 December 2016; pp. 153-158 [33] P. Khan, F.; Pi, Z. mmWave mobile broadband (MMB): Unleashing the $\mathrm{GHz}$ spectrum. In Proceedings of the 34th IEEE Sarnoff Symposium, Princeton, NJ, USA, 34 May 2011; pp. 1-6.

[34] M. Jameel, F.; Haider, M.A.A.; Butt, A.A. Robust localization in wireless sensor networks using RSSI. In Proceedings of the 2017 13th International Conference on Emerging Technologies (ICET), Islamabad, Pakistan, 2728 December 2017; pp. 1-6 